\title{
Enabling Grids for e-Science: The EGEE Project
}

\author{
Laure, Erwin CERN \\ et al
}

25 September 2008

Grid Computing: Infrastructure, Service, and Application. CRC Press

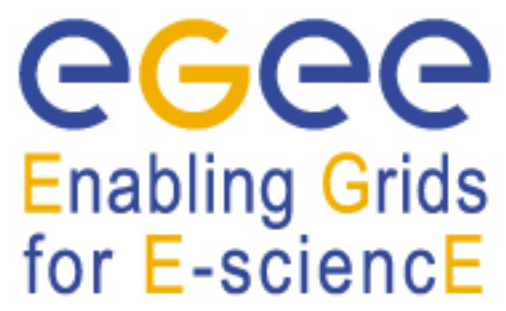

EGEE-II is a project funded by the European Commission

Contract number INFSO-RI-031688

The electronic version of this EGEE Publication is available on the CERN Document Server at the following URL:

<http://cdsweb. cern. ch/search. py?p=EGEE-PUB-2009-001> 


\title{
Enabling Grids for e-Science: The EGEE Project ${ }^{1}$
}

\author{
Erwin LAURE and Bob JONES \\ CERN, Geneva, Switzerland
}

\begin{abstract}
Enabling Grids for E-sciencE represents the worlds' largest multidisciplinary Grid infrastructure today. Co-funded by the European Commission, it brings together more than 250 resource centres from 48 countries to produce a reliable and scalable computing resource available to the European and global research community. This article provides an overview of EGEE, its infrastructure, middleware, applications and support structures. This article is intended to provide a first source of information for similar efforts elsewhere and based on EGEE's experiences a sustainable model for Grid operations will be discussed.
\end{abstract}

Keywords. Grid computing, e-Infrastructures, scientific computing

${ }^{1}$ This work is co-funded by the European Commission through the EGEE-II project, contract number INFSO-RI-031688. 